\title{
Multiple solutions of three-point boundary value problems for second-order impulsive differential equation at resonance
}

\section{Yulin Zhao ${ }^{* *}$, Haibo Chen ${ }^{2}$ and Qiming Zhang ${ }^{1}$}

\section{*Correspondence:}

zhaoylch@sina.com

'School of Science, Hunan

University of Technology, Zhuzhou,

Hunan 412007, PR China

Full list of author information is

available at the end of the article

\begin{abstract}
In this paper, by using the coincidence degree theory and the upper and lower solutions method, we deal with the existence of multiple solutions to three-point boundary value problems for second-order differential equation with impulses at resonance. An example is given to show the validity of our results.
\end{abstract}

Keywords: resonance; coincidence degree; upper and lower solutions; impulsive; three-point boundary value problems

\section{Introduction}

The purpose of the present paper is to investigate the following second-order impulsive differential equations:

$$
\left\{\begin{array}{l}
\left(\rho(t) u^{\prime}(t)\right)^{\prime}=f\left(t, u(t), u^{\prime}(t)\right), \quad t \in J, t \neq t_{k}, \\
\Delta u\left(t_{k}\right)=I_{k}\left(t_{k}, u\left(t_{k}\right)\right), \quad k=1,2, \ldots, m, \\
\Delta u^{\prime}\left(t_{k}\right)=J_{k}\left(t_{k}, u\left(t_{k}\right)\right),
\end{array}\right.
$$

together with the boundary conditions:

$$
u^{\prime}(0)=0, \quad u(1)=u(\eta)
$$

where $J=[0,1], \rho: J \rightarrow(0,+\infty)$ is a continuous differentiable function, $f: J \times R^{2} \rightarrow R$ is continuous, $0<\eta<1, I_{k}, J_{k} \in C(J, R)$ for $1 \leq k \leq m, m$ is a fixed positive integer, $0=t_{0}<$ $t_{1}<t_{2}<\cdots<t_{m}<t_{m+1}=1, \eta \neq t_{k}, \Delta u\left(t_{k}\right)=u\left(t_{k}^{+}\right)-u\left(t_{k}^{-}\right)$denotes the jump of $u(t)$ at $t=t_{k}$, $\Delta u^{\prime}\left(t_{k}\right)=u^{\prime}\left(t_{k}^{+}\right)-u^{\prime}\left(t_{k}^{-}\right) \cdot u^{\prime}\left(t_{k}^{+}\right), u\left(t_{k}^{+}\right)\left(u^{\prime}\left(t_{k}^{-}\right), u\left(t_{k}^{-}\right)\right)$represent the right limit (left limit) of $u^{\prime}(t)$ and $u(t)$ at $t=t_{k}$, respectively.

Impulsive differential equations describe processes which experience a sudden change of their state at certain moments. The theory of impulse differential equations has been a significant development in recent years and played a very important role in modern applied mathematical models of real processes rising in phenomena studied in physics, population dynamics, chemical technology, biotechnology, and economics; see [1-10] and the references therein. 
Recently, several authors (see $[6,11-14]$ and the references therein) have studied the existence of nontrivial or positive solutions for second-order three-point boundary value problem of the type

$$
\left\{\begin{array}{l}
u^{\prime \prime}=f\left(t, u, u^{\prime}\right), \\
u^{\prime}(0)=0, \quad u(1)=a u(\eta) .
\end{array}\right.
$$

Note that the nonlinear term $f$ depends on $u$ and its derivative $u^{\prime}$, then the relative problem becomes more complicated. A general method to deal with this difficulty is to add some conditions to restrict the growth of the $u^{\prime}$ term. One condition is the Caratheodory nonlinearity, the other usual condition is Nagumo condition or Nagumo-Winter condition (see $[2,9,12,15-20])$. When $a \neq 1$, the linear operator $L u=u^{\prime \prime}$ is invertible, this is the so-called non-resonance case. Gupta et al. made use of the Leray-Schauder continuation theorem to get the results on the existence of the solution for the problems (1.3) when $a \neq 1$ in [14]. By using the Leray-Schauder continuation theorem and in the presence of two pairs of upper and lower solutions, Khan and Webb [12] established the existence of at least three solutions for the problem (1.3) when $a \neq 1$. The linear operator $L u=u^{\prime \prime}$ is non-invertible when $a=1$, this is the so-called resonance case, and the Leray-Schauder continuation theorem cannot be applied. In [11], by using the coincidence degree theory of Mawhin [21] and some linear or non-linear growth assumptions on $f$, Feng and Webb obtained the existence of the solution of the problem (1.3) when $a=1$. By applying the nonlinear alternative of Leray-Schauder, Ma [13] have showed the existence of at least one solution for the problem (1.3) when $a=1$.

Recently, using the coincidence degree theory and the concept of autonomous curvature bound set, Liu and $\mathrm{Yu}$ [6] have studied the existence of at least one solution for the problem (1.1)-(1.2) when $\Delta u\left(t_{k}\right)=I_{k}\left(u\left(t_{k}\right), u^{\prime}\left(t_{k}\right)\right), \Delta u^{\prime}\left(t_{k}\right)=J_{k}\left(u\left(t_{k}\right), u^{\prime}\left(t_{k}\right)\right)$.

In the present paper, we assume that there exist $n(n \in N$ and $n \geq 2)$ pairs of upper and lower solutions for problem (1.1)-(1.2) and the nonlinear $f$ satisfies a Nagumo-like growth condition with respect to $u^{\prime}$. By considering a suitably modified nonlinearity and applying the coincidence degree method of Mawhin [21], the existence of multiple solutions for the problem (1.1)-(1.2) is given.

\section{Preliminaries}

Let

$$
X=P C^{1}(J) \cap\left\{u^{\prime}(0)=0, u(1)=u(\eta)\right\}, \quad Z=P C(J) \times R^{2 m},
$$

where

$$
\begin{aligned}
P C(J)= & \left\{u \in C\left(J^{*}\right), u\left(t^{-}\right) \text {and } u\left(t^{+}\right) \text {exist, and } u\left(t_{k}^{-}\right)=u\left(t_{k}\right)\right\} . \\
P C^{1}(J)= & \left\{u: J \rightarrow R: u(t) \text { is continuously differentiable for } t \neq 0,1, t_{k} ; u^{\prime}\left(t^{-}\right)\right. \\
& \text {and } \left.u^{\prime}\left(t^{+}\right) \text {exist, and } u^{\prime}\left(t_{k}^{-}\right)=u^{\prime}\left(t_{k}\right)\right\}, \quad J^{*}=J \backslash\left\{t_{1}, t_{2}, \ldots, t_{m}\right\} .
\end{aligned}
$$

Obviously, $X$ is a Banach space with the following norm:

$$
\|u\|_{X}=\max \left\{\sup _{t \in J}|u(t)|, \sup _{t \in J}\left|u^{\prime}(t)\right|\right\} .
$$


In the following, we recall the concept of strict upper and lower solutions for problem (1.1)-(1.2).

Definition 2.1 A function $\alpha(t) \in P C^{1}(J) \cap C^{2}\left(J^{*}\right)$ is said to be a strict lower solution of the problem (1.1)-(1.2) if

$$
\begin{aligned}
& \left(\rho(t) \alpha^{\prime}(t)\right)^{\prime}>f\left(t, \alpha(t), \alpha^{\prime}(t)\right), \quad t \in J^{*}, \\
& \Delta \alpha\left(t_{k}\right)=I_{k}\left(t_{k}, \alpha\left(t_{k}\right)\right), \quad \Delta \alpha^{\prime}\left(t_{k}\right) \geq J_{k}\left(t_{k}, \alpha\left(t_{k}\right)\right), \quad k=1,2, \ldots, m, \\
& \alpha^{\prime}(0) \geq 0, \quad \alpha(1)-\alpha(\eta) \leq 0 .
\end{aligned}
$$

Similarly, a function $\beta(t) \in P C^{1}(J) \cap C^{2}\left(J^{*}\right)$ is said to be a strict upper solution of the problem (1.1)-(1.2) if

$$
\begin{aligned}
& \left(\rho(t) \beta^{\prime}(t)\right)^{\prime}<f\left(t, \beta(t), \beta^{\prime}(t)\right), \quad t \in J^{\prime \prime}, \\
& \Delta \beta\left(t_{k}\right)=I_{k}\left(t_{k}, \beta\left(t_{k}\right)\right), \quad \Delta \beta^{\prime}\left(t_{k}\right) \leq J_{k}\left(t_{k}, \beta\left(t_{k}\right)\right), \quad k=1,2, \ldots, m, \\
& \beta^{\prime}(0) \leq 0, \quad \beta(1)-\beta(\eta) \geq 0 .
\end{aligned}
$$

Remark 2.2 Let $f: J \times R^{2} \rightarrow R$ be continuous, $I_{k}, J_{k} \in C(J, R)$, and $u \in P C^{1}(J) \cap C^{2}\left(J^{*}\right)$ is a solution of the problem (1.1)-(1.2), if $\alpha(\beta)$ is a strict lower solution (strict upper solution) for the problem (1.1)-(1.2) with $\alpha \leq u(u \leq \beta)$, then $\alpha<u(u<\beta)$ on $(0,1)$.

Definition 2.3 Let $\alpha$ be a strict lower solution and $\beta$ be a strict upper solution for the problem (1.1)-(1.2) satisfying $\alpha(t)<\beta(t)$ on $J$. We say that $f: J \times R^{2} \rightarrow R$ has property $(H)$ relative to $\alpha$ and $\beta$, if there exists a function $\psi \in C^{1}([0,+\infty),(0,+\infty))$ such that

$$
|f(t, u, p)|<\psi(|p|)
$$

for all $u(t) \in(-\beta(t),-\alpha(t)) \cup(\alpha(t), \beta(t)), t \in J$, and

$$
\int_{0}^{+\infty} \frac{s}{\theta s+\psi(s)} d s=+\infty
$$

where $0 \leq \theta<+\infty$ with $\left|\rho^{\prime}(t)\right| \leq \theta, t \in J$.

\section{The key lemmas}

Let $\operatorname{dom} L=C^{2}\left(U^{*}\right) \cap X$, and

$$
\begin{aligned}
L: \operatorname{dom} L \rightarrow Z, \quad & u \rightarrow\left(\left(\rho(t) u^{\prime}(t)\right)^{\prime}, \Delta u\left(t_{1}\right), \ldots, \Delta u\left(t_{m}\right), \Delta u^{\prime}\left(t_{1}\right), \ldots, \Delta u^{\prime}\left(t_{m}\right)\right), \\
N: u \rightarrow z, \quad u \rightarrow & \left(f\left(t, u, u^{\prime}\right), I_{1}\left(t_{1}, u\left(t_{1}\right)\right), \ldots, I_{m}\left(t_{m}, u\left(t_{m}\right)\right),\right. \\
& \left.J_{1}\left(t_{1}, u\left(t_{1}\right)\right), \ldots, J_{m}\left(t_{m}, u\left(t_{m}\right)\right)\right) .
\end{aligned}
$$

Then the problem (1.1)-(1.2) can be written as

$$
L u=N u, \quad u \in \operatorname{dom} L .
$$


Lemma 3.1 Suppose that $L$ be defined in the above. Then $L$ is a Fredholm operator of index zero. Furthermore

$$
\operatorname{Ker}(L)=\{u \in X: u=c, c \in R\},
$$

and

$$
\begin{aligned}
\operatorname{Im}(L)= & \left\{\left(y, a_{1}, \ldots, a_{m}, b_{1}, \ldots, b_{m}\right):\left(\rho(t) u^{\prime}(t)\right)^{\prime}=y(t), \Delta u\left(t_{k}\right)=a_{k}, \Delta u^{\prime}\left(t_{k}\right)=b_{k},\right. \\
& k=1, \ldots, m, \text { for some } y \in \operatorname{dom} L\} \\
= & \left\{\left(y, a_{1}, \ldots, a_{m}, b_{1}, \ldots, b_{m}\right): \int_{\eta}^{1} \frac{1}{\rho(s)} \int_{0}^{s} y(\tau) d \tau d s+\int_{\eta}^{1} \frac{1}{\rho(s)} \sum_{t_{k}<s} \rho\left(t_{k}\right) b_{k} d s\right. \\
& \left.+\sum_{\eta<t_{k}<1} a_{k}=0\right\} .
\end{aligned}
$$

Proof Firstly, it is clear that (3.1) holds. Next, we shall prove that (3.2) holds.

The following problem:

$$
\left\{\begin{array}{l}
\left(\rho(t) u^{\prime}(t)\right)^{\prime}=y(t), \\
\Delta u\left(t_{k}\right)=a_{k}, \quad \Delta u^{\prime}\left(t_{k}\right)=b_{k}, \quad k=1, \ldots, m
\end{array}\right.
$$

has a solution $u(t)$ satisfying $u^{\prime}(0)=0$ and $u(1)=u(\eta)$ if and only if

$$
\int_{\eta}^{1} \frac{1}{\rho(s)} \int_{0}^{s} y(\tau) d \tau d s+\int_{\eta}^{1} \frac{1}{\rho(s)} \sum_{t_{k}<s} \rho\left(t_{k}\right) b_{k} d s+\sum_{\eta<t_{k}<1} a_{k}=0 .
$$

In fact, if (3.3) has a solution $u(t)$ satisfying $u^{\prime}(0)=0, u(1)=u(\eta)$, then from (3.3) we have

$$
u(t)=u(0)+\int_{0}^{t} \frac{1}{\rho(s)} \int_{0}^{s} y(\tau) d \tau d s+\int_{0}^{t} \frac{1}{\rho(s)} \sum_{t_{k}<s} \rho\left(t_{k}\right) b_{k} d s+\sum_{t_{k}<t} a_{k} .
$$

According to $u^{\prime}(0)=0, u(1)=u(\eta)$, we get (3.4).

On the other hand, if (3.4) holds, setting

$$
u(t)=c+\int_{0}^{t} \frac{1}{\rho(s)} \int_{0}^{s} y(\tau) d \tau d s+\int_{0}^{t} \frac{1}{\rho(s)} \sum_{t_{k}<s} \rho\left(t_{k}\right) b_{k} d s+\sum_{t_{k}<t} a_{k},
$$

where $c$ is an arbitrary constant, then $u(t)$ is a solution of (3.3) with $u^{\prime}(0)=0, u(1)=u(\eta)$. Hence (3.2) holds.

Take the projector $Q: Z \rightarrow Z$ as follows:

$$
\begin{aligned}
& Q\left(y, a_{1}, \ldots, a_{m}, b_{1}, \ldots, b_{m}\right) \\
& =\left(\frac{1}{\phi(1)-\phi(\eta)}\left[\int_{\eta}^{1} \frac{1}{\rho(s)} \int_{0}^{s} y(\tau) d \tau d s+\int_{\eta}^{1} \frac{1}{\rho(s)} \sum_{t_{k}<s} \rho\left(t_{k}\right) b_{k} d s+\sum_{\eta<t_{k}<1} a_{k}\right],\right. \\
& \quad 0, \ldots, 0),
\end{aligned}
$$


where $\phi(t)=\int_{0}^{t} \frac{s}{\rho(s)} d s, t \in(0,1)$. For every $\left(y, a_{1}, \ldots, a_{m}, b_{1}, \ldots, b_{m}\right) \in Z$, set

$$
z=\left(y_{1}, a_{1}, \ldots, a_{m}, b_{1}, \ldots, b_{m}\right)=\left(y, a_{1}, \ldots, a_{m}, b_{1}, \ldots, b_{m}\right)-Q\left(y, a_{1}, \ldots, a_{m}, b_{1}, \ldots, b_{m}\right) .
$$

Thus, we obtain

$$
\begin{aligned}
\int_{\eta}^{1} & \frac{1}{\rho(s)} \int_{0}^{s} y_{1}(\tau) d \tau d s+\int_{\eta}^{1} \frac{1}{\rho(s)} \sum_{t_{k}<s} \rho\left(t_{k}\right) b_{k} d s+\sum_{\eta<t_{k}<1} a_{k} \\
= & {\left[\int_{\eta}^{1} \frac{1}{\rho(s)} \int_{0}^{s} y(\tau) d \tau d s+\int_{\eta}^{1} \frac{1}{\rho(s)} \sum_{t_{k}<s} \rho\left(t_{k}\right) b_{k} d s+\sum_{\eta<t_{k}<1} a_{k}\right] } \\
& \times\left[1-\frac{1}{\phi(1)-\phi(\eta)} \int_{\eta}^{1} \frac{d s}{\rho(s)}\right]=0 .
\end{aligned}
$$

Then $z \in \operatorname{Im} L$. Hence $Z=\operatorname{Im} L+R$. Since $\operatorname{Im} L \cap R=\{0\}$, we have $Z=\operatorname{Im} L \oplus R$, which implies $\operatorname{dim} \operatorname{Ker}(L)=\operatorname{dom} R=c o \operatorname{dim} \operatorname{Im} L=1$. Hence $L$ is a Fredholm operator of index zero.

Take $P: Z \rightarrow Z, P u=u(0)$. So the generalized inverse $K_{P}: \operatorname{Im} L \rightarrow \operatorname{dom} L \cap \operatorname{Ker} P$ of $L$ can be written as

$$
\begin{aligned}
K_{P} z(t) & =K_{P}\left(y, a_{1}, \ldots, a_{m}, b_{1}, \ldots, b_{m}\right) \\
& =\int_{0}^{t} \frac{1}{\rho(s)} \int_{0}^{s} y(\tau) d \tau d s+\int_{0}^{t} \frac{1}{\rho(s)} \sum_{t_{k}<s} \rho\left(t_{k}\right) b_{k} d s+\sum_{t_{k}<t} a_{k} .
\end{aligned}
$$

Set $\delta:=\min _{t \in J} \rho(t)>0, d \gg 1$. Then for the function $\psi$ defined by (2.8), let $h_{1}(u)$ be the solution of the following initial value problem:

$$
\delta y y^{\prime}+\theta y+\psi(y)=0, \quad y(0)=d
$$

and $h_{2}(u)$ be the solution of the following initial value problem:

$$
\delta y y^{\prime}-\theta y-\psi(y)=0, \quad y(0)=d
$$

Lemma 3.2 Suppose that there exists a constant $M>0$, then $h_{1}(u)$ is well defined in $[0, M]$ and positive on this interval, $h_{2}(u)$ is also well defined and positive in $[-M, 0]$. Moreover, if $d \gg 1$, then $h_{1}(u) \gg 1$ for any $u \in[0, M] ; h_{2}(u) \gg 1$ for any $u \in[-M, 0]$.

Proof We only consider the case $y=h_{1}(u)$ (in the case $y=h_{2}(u)$, the proof is similar). Assume that there exists a $u \in[0, M]$ such that $y(u)=h_{1}(u)=0$. Let $u_{0}=\inf \left\{u: h_{1}(u)=\right.$ $0, u \in[0, M]\}$. It follows from (3.7) that

$$
-\frac{y y^{\prime}}{\theta y+\psi(y)}=\delta^{-1}, \quad y>0
$$

Integrating the above equation over $\left[0, u_{0}\right]$ we get (let $\left.\tau=y(s)\right)$

$$
-\int_{0}^{u_{0}} \frac{y(s) y^{\prime}(s) d s}{\theta y(s)+\psi(y(s))}=\int_{0}^{d} \frac{\tau d \tau}{\theta \tau+\psi(\tau)}=\delta^{-1} u_{0} \leq \delta^{-1} M
$$


However, the left side of the above equation equals $\infty$ by (2.8). We reach a contradiction. Hence $h_{1}(u)>0$ for every $u \geq 0$. From $d \gg 1$, by the continuity of solution of differential equations on the initial values, we obtain $h_{1}(u) \gg 1$ for $u \in[0, M]$. The proof is complete.

Define the following sets:

$$
\begin{aligned}
G= & \left\{(t, u, p): t \in J,|u|<M,|p|<h_{1}(u) \text { for } u \in[0, M],\right. \\
& \text { and } \left.|p|<h_{2}(u) \text { for } u \in[-M, 0]\right\}, \\
\Omega= & \left\{u \in P C^{1}(J):\left(t, u(t), u^{\prime}(t)\right) \in G, t \in J ;\left(t_{k}^{+}, u\left(t_{k}^{+}\right), u^{\prime}\left(t_{k}^{+}\right)\right) \in G, k=1, \ldots, m\right\} .
\end{aligned}
$$

Define the function $h(u)$ as

$$
h(u)= \begin{cases}h_{1}(u), & u \in[0, M], \\ h_{2}(u), & u \in[-M, 0] .\end{cases}
$$

Lemma 3.3 Let Deg denote the coincidence degree. Let the following conditions hold:

(i) $f(t,-M, 0)<0<f(t, M, 0), \forall t \in J$ ( $M$ is given in Lemma 3.2);

(ii) $|f(t, u, p)|<\psi(|p|), \forall t \in J,|u| \leq M, p \in R$;

(iii) $I_{k}\left(t_{k}, \pm M\right)=0$, and $J_{k}\left(t_{k},-M\right)<0<J_{k}\left(t_{k}, M\right), k=1, \ldots, m$.

Then

$$
\operatorname{Deg}[(L, N), \Omega]=-1
$$

Proof Consider the following family of equations:

$$
L u=\lambda N u, \quad \lambda \in(0,1] .
$$

We will show

$$
L u \neq \lambda N u, \quad \forall u \in \partial \Omega, \lambda \in(0,1] .
$$

If not, then there exist some $\lambda \in(0,1]$ and $u \in \partial \Omega$ such that (3.10) holds. Note that $u \in \partial \Omega$ if and only if $\left(t, u(t), u^{\prime}(t)\right) \in \bar{G}$ and either $\left(\bar{t}, u(\bar{t}), u^{\prime}(\bar{t})\right) \in \partial G$ for some $\bar{t} \in J$, or $\left(t_{k_{0}}^{+}, u\left(t_{k_{0}}^{+}\right), u^{\prime}\left(t_{k_{0}}^{+}\right)\right) \in \partial \Omega$ for some $k_{0} \in\{1,2, \ldots, m\}$. There are two possibilities.

Case (I). If $\left(\bar{t}, u(\bar{t}), u^{\prime}(\bar{t})\right) \in \partial G, \bar{t} \in[0,1], \bar{t} \neq t_{k}^{+}$. In this case, $|u(\bar{t})|=M$, or $\left|u^{\prime}(\bar{t})\right|=h(u(\bar{t}))$.

Subcase (1). Suppose $|u(\bar{t})|=M$. Let $g(t)=\frac{1}{2}(u(t))^{2}-\frac{1}{2} M$. Then $g(t) \leq 0, t \in J$ and $g(\bar{t})=0$. When $t \leq \bar{t} \in(0,1), g(t) \leq 0$, we get

$$
0 \leq g^{\prime}(\bar{t}-0)=u(\bar{t}) u^{\prime}(\bar{t})
$$

If $g^{\prime}(\bar{t}-0)=0$, then $u^{\prime}(\bar{t})=0$. From condition (i), we have

$$
0 \geq g^{\prime \prime}(\bar{t}-0)=\left(u^{\prime}(\bar{t})\right)^{2}+\frac{u(\bar{t})}{\rho(\bar{t})} \cdot\left(\rho(\bar{t}) u^{\prime}(\bar{t})\right)^{\prime}= \pm \frac{M}{\rho(\bar{t})} \cdot \lambda f(\bar{t}, \pm M, 0)>0,
$$

which is a contradiction. 
If $g^{\prime}(\bar{t}-0)>0$, then $\bar{t}=t_{k_{0}}$ for some $k_{0} \in\{1, \ldots, m\}$ and $g^{\prime}\left(t_{k_{0}}-0\right)>0$. Thus from (iii), we have

$$
\begin{aligned}
g^{\prime}\left(t_{k_{0}}+0\right)= & {\left[u\left(t_{k_{0}}\right)+\lambda I_{k_{0}}\left(t_{k_{0}}, u\left(t_{k_{0}}\right)\right)\right] \cdot\left[u^{\prime}\left(t_{k_{0}}\right)+\lambda J_{k_{0}}\left(t_{k_{0}}, u\left(t_{k_{0}}\right)\right)\right] } \\
= & u\left(t_{k_{0}}\right) u^{\prime}\left(t_{k_{0}}\right)+u\left(t_{k_{0}}\right) \cdot \lambda J_{k_{0}}\left(t_{k_{0}}, u\left(t_{k_{0}}\right)\right)+u^{\prime}\left(t_{k_{0}}\right) \cdot \lambda I_{k_{0}}\left(t_{k_{0}}, u\left(t_{k_{0}}\right)\right) \\
& +\lambda^{2} I_{k_{0}}\left(t_{k_{0}}, u\left(t_{k_{0}}\right)\right) \cdot J_{k_{0}}\left(t_{k_{0}}, u\left(t_{k_{0}}\right)\right) \\
= & g^{\prime}\left(t_{k_{0}}-0\right)+\left[ \pm M \cdot \lambda J_{k_{0}}\left(t_{k_{0}}, \pm M\right)\right]>0 .
\end{aligned}
$$

On the other hand, $g(t) \leq 0, t \in J$ and $g\left(t_{k_{0}}+0\right)=0$, thus

$$
0 \geq g^{\prime}\left(t_{k_{0}}+0\right)=\left[u\left(t_{k_{0}}\right)+\lambda I_{k_{0}}\left(t_{k_{0}}, u\left(t_{k_{0}}\right)\right)\right] \cdot\left[u^{\prime}\left(t_{k_{0}}\right)+\lambda J_{k_{0}}\left(t_{k_{0}}, u\left(t_{k_{0}}\right)\right)\right]>0,
$$

which is a contradiction.

If $\bar{t}=0$, it is easy to see that $g(0)=0$. Since $u^{\prime}(0)=0$, we have $g^{\prime}(0)=0$, thus we can obtain $g^{\prime \prime}(0) \leq 0$. However, from condition (i) we know

$$
0 \geq \rho(0) g^{\prime \prime}(0)=u(0) \cdot \lambda f(0, u(0), 0)>0
$$

which is a contradiction.

If $\bar{t}=1$, then $|u(1)|=M$. This means that $u(1) \in \partial G$. Since $u(\eta)=u(1)$, we have $u(\eta) \in \partial G$. However, according to the above arguments, we know $u(\eta) \notin \partial G$, which is a contradiction.

Subcase (2). Suppose $\left|u^{\prime}(\bar{t})\right|=h(u(\bar{t}))$ for $\bar{t} \in\left(t_{k_{0}}, t_{k_{0}+1}\right], k_{0} \in\{0,1, \ldots, m\}$. Obviously, $\bar{t} \neq 0$. Since $\left|(\rho(t) u(t))^{\prime}\right|=\left|f\left(t, u(t), u^{\prime}(t)\right)\right|<\psi\left(\left|u^{\prime}(t)\right|\right)$. Thus we get

$$
\begin{aligned}
& \rho(t)\left|u^{\prime \prime}(t)\right|-\left|\rho^{\prime}(t)\right|\left|u^{\prime}(t)\right|<\psi\left(\left|u^{\prime}(t)\right|\right), \\
& \quad\left|u^{\prime \prime}(t)\right|<\frac{1}{\delta}\left[\left|\rho^{\prime}(t)\right|\left|u^{\prime}(t)\right|+\psi\left(\left|u^{\prime}(t)\right|\right)\right] \leq \frac{1}{\delta}\left[\theta\left|u^{\prime}(t)\right|+\psi\left(\left|u^{\prime}(t)\right|\right)\right] .
\end{aligned}
$$

Let

$$
p(t)=\frac{1}{2}\left(u^{\prime}(\bar{t})\right)^{2}-\frac{1}{2}(h(u(t)))^{2} .
$$

Without loss of generality, we suppose that $u^{\prime}(\bar{t})=h(u(\bar{t}))>0$ for $\bar{t} \in\left(t_{k_{0}}, t_{k_{0}+1}\right]$. Then we have three possibilities.

(i) If $u(\bar{t})=0$, then there exists a sufficiently small neighborhood of $\bar{t}$ such that $u(t)>0$. Thus $p(t)=\frac{1}{2}\left(u^{\prime}(t)\right)^{2}-\frac{1}{2}\left(h_{1}(u(t))\right)^{2}$, and $p(t)$ has a local maximum value on $\bar{t}$, which implies $p^{\prime}(\bar{t}+0) \leq 0$. But in this case, from (3.7), (3.12), and (ii), we have

$$
\begin{aligned}
p^{\prime}(\bar{t}+0) & =u^{\prime}(\bar{t}) \cdot\left[u^{\prime \prime}(\bar{t})-h_{1}(u(\bar{t})) h_{1}^{\prime}(u(\bar{t}))\right] \\
& >u^{\prime}(\bar{t}) \cdot\left[-\frac{1}{\delta}\left(\theta u^{\prime}(\bar{t})+\psi\left(\left|u^{\prime}(\bar{t})\right|\right)\right)+\frac{\theta h_{1}(u(t))+\psi\left(h_{1}(u(t))\right)}{\delta}\right]=0,
\end{aligned}
$$

which is a contradiction.

(ii) If $u(\bar{t})<0$, then there exists a sufficiently small neighborhood of $\bar{t}$ such that $u(t)<0$. Thus $p(t)=\frac{1}{2}\left(u^{\prime}(t)\right)^{2}-\frac{1}{2}\left(h_{2}(u(t))\right)^{2}$, and $p(t)$ has a local maximum value on $\bar{t}$, which implies 
$p^{\prime}(\bar{t}) \geq 0$. But in this case, from (3.8), (3.12), and (ii), we have

$$
\begin{aligned}
p^{\prime}(\bar{t}) & =u^{\prime}(\bar{t}) \cdot\left[u^{\prime \prime}(\bar{t})-h_{2}(u(\bar{t})) h_{2}^{\prime}(u(\bar{t}))\right] \\
& <u^{\prime}(\bar{t}) \cdot\left[\frac{1}{\delta}\left(\theta u^{\prime}(\bar{t})+\psi\left(\left|u^{\prime}(\bar{t})\right|\right)\right)-\frac{\theta h_{2}(u(t))+\psi\left(h_{2}(u(t))\right)}{\delta}\right]=0,
\end{aligned}
$$

which is a contradiction.

(iii) If $u(\bar{t})>0$, then there exists a sufficiently small neighborhood of $\bar{t}$ such that $u(t)>0$. Thus $p(t)=\frac{1}{2}\left(u^{\prime}(t)\right)^{2}-\frac{1}{2}\left(h_{1}(u(t))\right)^{2}$. By the same argument as in (3.13), we reach a contradiction.

Case (II). $\left(t_{k_{0}}^{+}, u\left(t_{k_{0}}^{+}\right), u^{\prime}\left(t_{k_{0}}^{+}\right)\right) \in \partial \Omega$ for some $k_{0} \in\{1,2, \ldots, m\}$. In this case, $\left|u\left(t_{k_{0}}^{+}\right)\right|=M$, or $\left|u^{\prime}\left(t_{k_{0}}^{+}\right)\right|=h\left(u\left(t_{k_{0}}^{+}\right)\right)$.

Subcase (3). Suppose $\left|u\left(t_{k_{0}}^{+}\right)\right|=M$ for some $k_{0} \in\{1,2, \ldots, m\}$. Let $g(t)$ be defined in the above. Obviously, we have $g(t) \leq 0$ for $t \in J$ and $g\left(t_{k_{0}}+0\right)=0$. It follows from (iii) that

$$
\begin{aligned}
0 & \geq g^{\prime}\left(t_{k_{0}}+0\right)=\left[u\left(t_{k_{0}}\right)+\lambda I_{k_{0}}\left(t_{k_{0}}, u\left(t_{k_{0}}\right)\right)\right] \cdot\left[u^{\prime}\left(t_{k_{0}}\right)+\lambda J_{k_{0}}\left(t_{k_{0}}, u\left(t_{k_{0}}\right)\right)\right] \\
& =u\left(t_{k_{0}}\right) u^{\prime}\left(t_{k_{0}}\right)+u\left(t_{k_{0}}\right) \cdot \lambda J_{k_{0}}\left(t_{k_{0}}, u\left(t_{k_{0}}\right)\right) .
\end{aligned}
$$

If $g^{\prime}\left(t_{k_{0}}+0\right)=0$, then $u^{\prime}\left(t_{k_{0}}^{+}\right)=0$. Form (3.11) and (i), we have

$$
0 \geq g^{\prime \prime}\left(t_{k_{0}}+0\right)=\left(u^{\prime}\left(t_{k_{0}}^{+}\right)\right)^{2}+\frac{u\left(t_{k_{0}}^{+}\right)}{\rho\left(t_{k_{0}}^{+}\right)} \cdot\left(\rho\left(t_{k_{0}}\right) u^{\prime}\left(t_{k_{0}}^{+}\right)\right)^{\prime}= \pm \frac{M}{\rho\left(t_{k_{0}}^{+}\right)} \cdot \lambda f\left(t_{k_{0}}, \pm M, 0\right)>0,
$$

which is a contradiction.

If $g^{\prime}\left(t_{k_{0}}+0\right)<0$, together with (iii), we get $g^{\prime}\left(t_{k_{0}}-0\right)=u\left(t_{k_{0}}\right) u^{\prime}\left(t_{k_{0}}\right)<0$. But $g\left(t_{k_{0}}\right)=0$ and $g(t) \leq 0$ for $t \in J$, which yields $g^{\prime}\left(t_{k_{0}}-0\right)>0$, and we reach a contradiction.

Similar to subcase (2), we can show that $\left|u^{\prime}\left(t_{k_{0}}^{+}\right)\right|=h\left(u\left(t_{k_{0}}^{+}\right)\right)$is also impossible. Combining the results of case (I) and case (II) we obtain (3.11).

On the other hand, for $\lambda \in(0,1]$, it follows from [21] that (3.10) is equivalent to the following family of operator equations:

$$
u=P u+Q N u+\lambda K_{P}(E-Q) N u,
$$

where $E$ is the identity mapping.

Note that $\operatorname{Ker} L=R$, and

$$
\Omega \cap \operatorname{Ker} L=\left\{c \in R:(t, c, p) \in G, t \in J ;\left(t_{k}^{+}, c, p\right) \in G \text {, for some } k=1, \ldots, m\right\} .
$$

From (3.5) and (3.6), we have

$$
\begin{aligned}
Q N u= & \left(\frac { 1 } { \phi ( 1 ) - \phi ( \eta ) } \left[\int_{\eta}^{1} \frac{1}{\rho(s)} \int_{0}^{s} f\left(\tau, u(\tau), u^{\prime}(\tau)\right) d \tau d s\right.\right. \\
& \left.\left.+\int_{\eta}^{1} \frac{1}{\rho(s)} \sum_{t_{k}<s} \rho\left(t_{k}\right) J_{k}\left(t_{k}, u\left(t_{k}\right)\right) d s+\sum_{\eta<t_{k}<1} I_{k}\left(t_{k}, u\left(t_{k}\right)\right)\right], 0, \ldots, 0\right),
\end{aligned}
$$


and

$$
\begin{aligned}
K_{P}(E-Q) N u= & \frac{1}{\phi(1)-\phi(\eta)}\left[\int_{0}^{t} \frac{1}{\rho(s)} \int_{0}^{s} f\left(\tau, u(\tau), u^{\prime}(\tau)\right) d \tau d s\right. \\
& \left.+\int_{0}^{t} \frac{1}{\rho(s)} \sum_{t_{k}<s} \rho\left(t_{k}\right) J_{k}\left(t_{k}, u\left(t_{k}\right)\right) d s+\sum_{t_{k}<t} I_{k}\left(t_{k}, u\left(t_{k}\right)\right)\right] \\
& -\frac{\phi(t)}{\phi(1)-\phi(\eta)}\left[\int_{\eta}^{1} \frac{1}{\rho(s)} \int_{0}^{s} f\left(\tau, u(\tau), u^{\prime}(\tau)\right) d \tau d s\right. \\
& \left.+\int_{\eta}^{1} \frac{1}{\rho(s)} \sum_{t_{k}<s} \rho\left(t_{k}\right) J_{k}\left(t_{k}, u\left(t_{k}\right)\right) d s+\sum_{\eta<t_{k}<1} I_{k}\left(t_{k}, u\left(t_{k}\right)\right)\right] .
\end{aligned}
$$

By the Ascoli-Arzela theorem, it is easy to show that $Q N(\bar{\Omega})$ is bounded and $K_{P}(E-Q) N$ : $\bar{\Omega} \rightarrow X$ is compact. Thus $N$ is $L$-compact on $\bar{\Omega}$.

Define a mapping $H: \bar{\Omega} \times[0,1] \rightarrow X$,

$$
H(u, \lambda)=P u+Q N u+\lambda K_{P}(E-Q) N u, \quad \forall(u, \lambda) \in \bar{\Omega} \times[0,1] .
$$

Then it is easy to prove that $H(u, \lambda)$ is completely continuous and we claim that

$$
u=H(u, \lambda), \quad \forall u \in \partial \Omega, 0 \leq \lambda \leq 1 .
$$

In fact, for $0<\lambda \leq 1$, it follows from (3.10) and (3.14) that (3.15) holds. For $\lambda=0$, if there exists a $\bar{u} \in \partial \Omega$ such that $\bar{u}=H(\bar{u}, 0)$, that is $\bar{u}=P \bar{u}+Q N \bar{u}$, in this case, $Q N \bar{u}=0, \bar{u} \in \operatorname{Ker} L$, hence $\bar{u}=M$ or $\bar{u}=-M$. However, it follows from (iii) that

$$
\begin{aligned}
Q N(c)= & \frac{1}{\phi(1)-\phi(\eta)}\left[\int_{\eta}^{1} \frac{1}{\rho(s)} \int_{0}^{s} f(\tau, c, 0) d \tau d s\right. \\
& \left.+\int_{\eta}^{1} \frac{1}{\rho(s)} \sum_{t_{k}<s} \rho\left(t_{k}\right) J_{k}\left(t_{k}, c\right) d s+\sum_{\eta<t_{k}<1} I_{k}\left(t_{k}, c\right)\right] \\
= & \frac{1}{\phi(1)-\phi(\eta)} \int_{\eta}^{1} \frac{1}{\rho(s)} \int_{0}^{s} f(\tau, c, 0) d \tau d s+\int_{\eta}^{1} \frac{1}{\rho(s)} \sum_{t_{k}<s} \rho\left(t_{k}\right) J_{k}\left(t_{k}, c\right) d s, \quad c \in R .
\end{aligned}
$$

Thus we get $Q N(M)>0$ and $Q N(-M)<0$, which contradicts $u=P u+Q N u$ for $u \in \operatorname{Ker} L$. Therefore (3.15) holds. As follows from [21] and by using the invariance of Leray-Schauder degree under homotopy, we obtain

$$
\begin{aligned}
\operatorname{Deg}(E-H(\cdot, 1), \Omega, 0) & =\operatorname{Deg}(E-H(\cdot, 0), \Omega, 0) \\
& =\operatorname{Deg}_{B}\left(\left.(E-P-Q N)\right|_{\operatorname{Ker} L \cap \bar{\Omega}}, \operatorname{Ker} L \cap \Omega, 0\right) \\
& =\operatorname{Deg}_{B}\left(\left.(-Q N)\right|_{\operatorname{Ker} L \cap \bar{\Omega}}, \operatorname{Ker} L \cap \Omega, 0\right) .
\end{aligned}
$$

Since $\operatorname{Ker} L$ is one dimensional and $Q N(M)>0, Q N(-M)<0$, we get

$$
\operatorname{Deg}_{B}\left(\left.(-Q N)\right|_{\operatorname{Ker} L \cap \bar{\Omega}}, \operatorname{Ker} L \cap \Omega, 0\right)=-1 .
$$

From the property of coincidence degree we proved Lemma 3.3. 


\section{Lemma 3.4 Assume that}

(c1) there exist lower and upper solutions $\alpha(t), \beta(t)$ of the problem (1.1)-(1.2), respectively, with $\alpha(t)<\beta(t)$;

(c2) $f: J \times R^{2} \rightarrow R$ is continuous and has property $(H)$ relative to $\alpha, \beta$;

(c3) $I_{k}, J_{k}$ are continuous for each $k=1,2, \ldots, m$, and satisfy

$$
I_{k}\left(t_{k}, \alpha\left(t_{k}\right)\right)=I_{k}\left(t_{k}, \beta\left(t_{k}\right)\right)=0, \quad \text { and } \quad J_{k}\left(t_{k}, \alpha\left(t_{k}\right)\right)<0<J_{k}\left(t_{k}, \beta\left(t_{k}\right)\right) \text {. }
$$

Then $\operatorname{Deg}\left[(L, N), \Omega_{\alpha \beta}\right]=-1$.

Proof Choose $M>0$ large enough for $u, p \in R, u \in(-\beta(t),-\alpha(t)) \cup(\alpha(t), \beta(t)), t \in J$, such that

$$
\begin{aligned}
& f(t, \beta(t), 0)+M-\beta(t)>0, \quad M>\beta(t), \forall t \in J, \\
& f(t, \alpha(t), 0)-M-\alpha(t)<0, \quad-M<\alpha(t), \forall t \in J, \\
& J_{k}\left(t_{k}, \alpha\left(t_{k}\right)\right)-M-\alpha\left(t_{k}\right)<0<J_{k}\left(t_{k}, \beta\left(t_{k}\right)\right)+M-\beta\left(t_{k}\right), \quad k=1, \ldots, m,
\end{aligned}
$$

and let $h_{i}(u)(i=1,2)$ be defined in (3.7) and (3.8), then it follows from Lemma 3.2 that we can choose $d>0$ large enough such that

$$
\begin{aligned}
& \min _{[0, M]} h_{1}(u)>\max \left\{\max _{t \in J}\left|\beta^{\prime}(t)\right|, \max _{t \in J}\left|\alpha^{\prime}(t)\right|\right\}, \\
& \min _{[-M, 0]} h_{2}(u)>\max \left\{\max _{t \in J}\left|\beta^{\prime}(t)\right|, \max _{t \in J}\left|\alpha^{\prime}(t)\right|\right\} .
\end{aligned}
$$

Define a set $\Omega_{\alpha \beta}$ as

$$
\Omega_{\alpha \beta}=\left\{u \in P C^{1}(J)|(t, u, p): \alpha(t)<u<\beta(t),| p \mid<h(u), \forall t \in J\right\},
$$

where $h(u)$ is given in (3.9).

Define the auxiliary functions $F$ and $\bar{I}_{k}, \bar{J}_{k}$ as follows:

$$
\begin{aligned}
& F(t, u, p)=f(t, n(t, u), q(t, p))+u(t)-n(t, u), \quad t \in J^{*}, \\
& \bar{I}_{k}\left(t_{k}, u\left(t_{k}\right)\right)=I_{k}\left(t_{k}, n\left(t_{k}, u\left(t_{k}\right)\right)\right), \quad k \in\{1, \ldots, m\}, \\
& \bar{J}_{k}\left(t_{k}, u\left(t_{k}\right)\right)=J_{k}\left(t_{k}, n\left(t_{k}, u\left(t_{k}\right)\right)\right)+u\left(t_{k}\right)-n\left(t_{k}, u\left(t_{k}\right)\right), \quad k \in\{1, \ldots, m\},
\end{aligned}
$$

where

$$
\begin{gathered}
n(t, u(t))= \begin{cases}\beta(t), & \beta(t)<u \leq M, \\
u(t), & \alpha(t) \leq u \leq \beta(t), \\
\alpha(t), & -M \leq u<\alpha(t),\end{cases} \\
q(t, p)= \begin{cases}h(u), & p>h(u),|u| \leq M, \\
p, & |p| \leq h(u),|u| \leq M, \\
-h(u), & p<-h(u),|u| \leq M .\end{cases}
\end{gathered}
$$


We then generalize $F$ to $J \times R^{2}$ and $\bar{I}_{k}, \bar{J}_{k}: J \times R \rightarrow R$. It is easy to see that $F, \bar{I}_{k}, \bar{J}_{k}$ are continuous and satisfy

$$
\begin{aligned}
& F(t,-M, 0)<0<F(t, M, 0), \\
& \bar{I}_{k}\left(t_{k}, \pm M\right)=0, \quad \text { and } \quad \bar{J}_{k}\left(t_{k},-M\right)<0<\bar{J}_{k}\left(t_{k},+M\right), \quad k \in\{1, \ldots, m\} .
\end{aligned}
$$

Moreover, when $|u| \leq M, F, \bar{I}_{k}, \bar{J}_{k}$ are bounded. It follows from Lemma 3.3 that

$$
\operatorname{Deg}[(L, \bar{N}), \Omega]=-1,
$$

where

$$
\bar{N} u=\left(F\left(t, u, u^{\prime}\right), \bar{I}_{1}\left(t_{1}, u\left(t_{1}\right)\right), \ldots, \bar{I}_{m}\left(t_{m}, u\left(t_{m}\right)\right), \bar{J}_{1}\left(t_{1}, u\left(t_{1}\right)\right), \ldots, \bar{J}_{m}\left(t_{m}, u\left(t_{m}\right)\right)\right) .
$$

Next we show

$$
\operatorname{Deg}\left[(L, \bar{N}), \Omega_{\alpha \beta}\right]=-1
$$

It suffices to show that

$$
L u=\bar{N} u, \quad \forall u \in \bar{\Omega} \backslash \Omega_{\alpha \beta} .
$$

In fact, let $u \in \bar{\Omega}$ such that $L u=\bar{N} u$ and assume that

$$
\max _{t \in J}\{u(t)-\beta(t)\}=u(\tau)-\beta(\tau) \geq 0 .
$$

Case (1). If there exists $\tau \in(0,1), \tau \neq t_{k}$ such that the function $y(t)=u(t)-\beta(t)$ attains its maximum value $y(\tau) \geq 0$, which implies $y^{\prime}(\tau)=0$ and $y^{\prime \prime}(\tau) \leq 0$. But on the other hand,

$$
\begin{aligned}
\rho(\tau) y^{\prime \prime}(\tau) & =\left(\rho(\tau) u^{\prime}(\tau)\right)^{\prime}-\left(\rho(\tau) \beta^{\prime}(\tau)\right)^{\prime} \\
& =F\left(\tau, \beta(\tau), u^{\prime}(\tau)\right)+u(\tau)-\beta(\tau)-\left(\rho(\tau) \beta^{\prime}(\tau)\right)^{\prime} \\
& =f\left(\tau, \beta(\tau), \beta^{\prime}(\tau)\right)+u(\tau)-\beta(\tau)-\left(\rho(\tau) \beta^{\prime}(\tau)\right)^{\prime}>0,
\end{aligned}
$$

which implies $y^{\prime \prime}(\tau)>0$. We reach a contradiction.

If $\tau=0$, then $y(0) \geq 0, y^{\prime}(0) \leq 0$. On the other hand, since $u^{\prime}(0)=0$ and $\beta(t)$ is a strict upper solution of problem (1.1)-(1.2), we see that $y^{\prime}(0)=u^{\prime}(0)-\beta^{\prime}(0) \geq 0$. Thus $y^{\prime}(0)=0$. Note that $y(t)$ assumes the maximum value at $t=0$, there exists $\tau_{0} \in(0,1), \tau_{0} \neq t_{k}$ such that $y\left(\tau_{0}\right) \geq 0$ and $y^{\prime \prime}\left(\tau_{0}\right) \leq 0$. By the same argument as in (3.18) where $\tau=\tau_{0}$, we reach a contradiction.

If $\tau=1$, then $y(1)=u(1)-\beta(1) \geq 0$. According to (2.6), we get

$$
y(1)=u(1)-\beta(1) \leq u(1)-\beta(\eta)=u(\eta)-\beta(\eta)=y(\eta),
$$

if $y(\eta)>y(1)$, which is a contradiction. Thus $y(\eta)=y(1)$, which implies that $y(t)$ also attains its maximum value at $t=\eta$. By the same argument as in (3.18) where $\tau=\eta$, we reach a contradiction. 
Hence the function $y(t)$ cannot have any nonnegative maximum value on the interval $(0,1), t \neq t_{k}$ for $k \in\{0,1, \ldots, m\}$.

Case (2). If there exists a $\tau \in J$ such that $y(\tau)=u(\tau)-\beta(\tau)=\varepsilon \geq 0$, from case (1), we get $\tau=t_{k}$ for some $k=1,2, \ldots, m$. Hence $n\left(t_{k}, u\left(t_{k}\right)\right)=\beta\left(t_{k}\right), \beta^{\prime}\left(t_{k}\right) \leq u^{\prime}\left(t_{k}\right)$, and consequently we have $y\left(t_{k}^{+}\right)=y\left(t_{k}\right)=\varepsilon$, which implies $y^{\prime}\left(t_{k}^{+}\right) \geq 0$, because

$$
\begin{aligned}
u^{\prime}\left(t_{k}^{+}\right)-u^{\prime}\left(t_{k}\right) & =\bar{J}_{k}\left(t_{k}, u\left(t_{k}\right)\right)=J_{k}\left(t_{k}, \beta\left(t_{k}\right)\right)+u\left(t_{k}\right)-\beta\left(t_{k}\right) \\
& \geq J_{k}\left(t_{k}, \beta\left(t_{k}\right)\right) \geq \beta^{\prime}\left(t_{k}^{+}\right)-\beta^{\prime}\left(t_{k}\right) .
\end{aligned}
$$

Consequently, we get $u^{\prime}\left(t_{k}^{+}\right)=\beta^{\prime}\left(t_{k}^{+}\right)$or $y^{\prime}\left(t_{k}^{+}\right)=0$ and $D_{+} y^{\prime}\left(t_{k}^{+}\right) \leq 0$.

By the continuity of $f$ and $\beta(t)$ is a strict upper solution of problem (1.1)-(1.2), there exists a sequence $\zeta_{j} \in R$ with $\zeta_{j}>0, \zeta_{j} \rightarrow 0$, as $j \rightarrow \infty$ such that

$$
\begin{aligned}
D^{+} \rho\left(t_{k}\right) \beta^{\prime}\left(t_{k}^{+}\right) & =\lim _{j \rightarrow \infty} \sup \frac{\rho\left(t_{k}+\zeta_{j}\right) \beta^{\prime}\left(t_{k}+\zeta_{j}\right)-\rho\left(t_{k}\right) \beta^{\prime}\left(t_{k}\right)}{\zeta_{j}} \\
& =\lim _{j \rightarrow \infty}\left(\rho\left(t_{k}+\zeta_{j}\right) \beta^{\prime}\left(t_{k}+\zeta_{j}\right)\right)^{\prime} \\
& <\lim _{j \rightarrow \infty}\left[f\left(t_{k}+\zeta_{j}, \beta\left(t_{k}+\zeta_{j}\right), \beta^{\prime}\left(t_{k}+\zeta_{j}\right)\right)\right]=f\left(t_{k}, \beta\left(t_{k}^{+}\right), \beta^{\prime}\left(t_{k}^{+}\right)\right),
\end{aligned}
$$

where $\zeta_{j} \in\left(t_{k}, t_{k}+\zeta_{j}\right)$ are from the mean value theorem. As before, we also get

$$
D_{+} \rho\left(t_{k}\right) u^{\prime}\left(t_{k}^{+}\right)=F\left(t_{k}, u\left(t_{k}^{+}\right), u^{\prime}\left(t_{k}^{+}\right)\right)
$$

where $D_{+} \rho\left(t_{k}\right) u^{\prime}\left(t_{k}^{+}\right)=\lim _{j \rightarrow \infty} \inf \frac{\rho\left(t_{k}+\zeta_{j}\right) u^{\prime}\left(t_{k}+\zeta_{j}\right)-\rho\left(t_{k}\right) u^{\prime}\left(t_{k}\right)}{\zeta_{j}}$. As a result, we can obtain

$$
D^{+} \rho\left(t_{k}\right) \beta^{\prime}\left(t_{k}^{+}\right) \geq D_{+} \rho\left(t_{k}\right) u^{\prime}\left(t_{k}^{+}\right)=f\left(t_{k}, \beta\left(t_{k}^{+}\right), \beta^{\prime}\left(t_{k}^{+}\right)\right)+y\left(t_{k}^{+}\right) \geq f\left(t_{k}, \beta\left(t_{k}^{+}\right), \beta^{\prime}\left(t_{k}^{+}\right)\right)
$$

which is a contradiction to (3.19).

Case (3). If $y(t)=u(t)-\beta(t)<\varepsilon$ for all $t \in J$, then there must be a $k_{0}\left(1 \leq k_{0} \leq m\right)$ such that $y\left(t_{k_{0}}^{+}\right)=u\left(t_{k_{0}}^{+}\right)-\beta\left(t_{k_{0}}^{+}\right)=\varepsilon$, and $y\left(t_{k_{0}}\right)<\varepsilon$, which implies $\beta\left(t_{k_{0}}^{+}\right)-\beta\left(t_{k_{0}}\right)<u\left(t_{k_{0}}^{+}\right)-u\left(t_{k_{0}}\right)$. Namely, $\Delta \beta\left(t_{k_{0}}\right)<\Delta u\left(t_{k_{0}}\right)$. However, this is impossible because

$$
\Delta u\left(t_{k_{0}}\right)=\bar{I}_{k_{0}}\left(t_{k_{0}}, n\left(t_{k_{0}}, u\left(t_{k_{0}}\right)\right)\right)=I_{k_{0}}\left(t_{k_{0}}, \beta\left(t_{k_{0}}\right)\right)=\Delta \beta\left(t_{k_{0}}\right) .
$$

Thus we have proved that $u(t)<\beta(t)$ on $J$. Similarly we can show that $\alpha(t)<u(t)$ on $J$. It then follows that $\alpha(t)<u(t)<\beta(t)$ on $J$.

We now shall prove that

$$
\left|u^{\prime}(t)\right|<h(u(t)) \quad \text { for } t \in\left[t_{k}, t_{k+1}\right], k=0,1, \ldots, m \text {. }
$$

Assume that (3.20) cannot hold. Thus there are two possibilities:

(a) There exists $\tau \in\left(t_{k_{0}}, t_{k_{0}+1}\right]$ for some $k_{0} \in\{0,1, \ldots, m\}$ such that $\left|u^{\prime}(\tau)\right| \geq h(u(\tau))$.

(b) There exists some $k_{0} \in\{0,1, \ldots, m\}$ such that $\left|u^{\prime}\left(t_{k_{0}}^{+}\right)\right| \geq h\left(u\left(t_{k_{0}}^{+}\right)\right)$.

For case (a), we assume $\max _{t \in\left(t_{k_{0}}, t_{k_{0}+1}\right]}\left\{u^{\prime}(t)-h(u(t))\right\}=\max _{t \in\left(t_{k_{0}}, t_{k_{0}+1}\right]} r(t)=u^{\prime}(\tau)-$ $h(u(\tau)) \geq 0$. 
Since $\left|(\rho(t) u(t))^{\prime}\right|=\left|F\left(t, u(t), u^{\prime}(t)\right)\right|=|f(t, u(t), h(u(t)))|<\psi(h(u(t)))$. Thus we have

$$
\begin{aligned}
& \rho(t)\left|u^{\prime \prime}(t)\right|-\left|\rho^{\prime}(t)\right|\left|u^{\prime}(t)\right|<\psi(h(u(t))), \\
& \quad\left|u^{\prime \prime}(t)\right|<\frac{1}{\delta}\left[\left|\rho^{\prime}(t)\right|\left|u^{\prime}(t)\right|+\psi(h(u(t)))\right] \leq \frac{1}{\delta}\left[\theta\left|u^{\prime}(t)\right|+\psi(h(u(t)))\right] .
\end{aligned}
$$

If $u(\tau)=0$, then there exists a sufficiently small neighborhood of $\tau$ such that $u(t)>0$. Thus $r(t)=u^{\prime}(t)-h_{1}(u(t))$ and $r^{\prime}(\tau+0) \leq 0$. However, it follows from (3.7) and (3.21) that

$$
\begin{aligned}
0 & \geq r^{\prime}(\tau+0)=u^{\prime \prime}(\tau)-h_{1}^{\prime}(u(\tau)) u^{\prime}(\tau) \\
& >-\frac{1}{\delta}\left[\theta u^{\prime}(\tau)+\psi\left(h_{1}(u(\tau))\right)\right]+\frac{\psi\left(h_{1}(u(\tau))\right)+\theta h_{1}(u(\tau))}{\delta h_{1}(u(\tau))} \cdot u^{\prime}(\tau) \\
& =\frac{\psi\left(h_{1}(u(\tau))\right)}{\delta h_{1}(u(\tau))}\left[u^{\prime}(\tau)-h_{1}(u(\tau))\right] \geq 0,
\end{aligned}
$$

which is a contradiction.

If $u(\tau)<0$, then there exists a sufficiently small neighborhood of $\tau$ such that $u(t)<0$. Thus $r(t)=u^{\prime}(t)-h_{2}(u(t))$ and $r^{\prime}(\tau)=0$. However, it follows from (3.8) and (3.21) that

$$
\begin{aligned}
0 & =r^{\prime}(\tau)=u^{\prime \prime}(\tau)-h_{2}^{\prime}(u(\tau)) u^{\prime}(\tau) \\
& <\frac{1}{\delta}\left[\theta u^{\prime}(\tau)+\psi\left(h_{1}(u(\tau))\right)\right]-\frac{\psi\left(h_{2}(u(\tau))\right)+\theta h_{2}(u(\tau))}{\delta h_{2}(u(\tau))} \cdot u^{\prime}(\tau) \\
& =-\frac{\psi\left(h_{2}(u(\tau))\right)}{\delta h_{2}(u(\tau))}\left[u^{\prime}(\tau)-h_{2}(u(\tau))\right] \leq 0,
\end{aligned}
$$

a contradiction.

If $u(\tau)>0$, then there exists a sufficiently small neighborhood of $\tau$ such that $u(t)>0$. Thus $r(t)=u^{\prime}(t)-h_{1}(u(t))$ and $r^{\prime}(\tau)=0$. By the similarly argument as in (3.22), we reach a contradiction.

Hence $u^{\prime}(t)<h(u(t)), t \in\left(t_{k}, t_{k+1}\right], k=0,1, \ldots, m$. Similarly, we can prove $-h(u(t))<u^{\prime}(t)$, $t \in\left(t_{k}, t_{k+1}\right], k=0,1, \ldots, m$.

Likewise, we can show that case (b) is also impossible, and thus (3.20) holds. Combining the above results, we see that if $u \in \Omega, L u=\bar{N} u$, then $u \in \Omega_{\alpha \beta}$. Hence (3.17) is proved. From the property of coincidence, we know that (3.16) holds. Since in $\Omega_{\alpha \beta}, F=f$, we have $N=\bar{N}$. The proof is complete.

\section{Main results}

We are now in a position to prove our main result on the existence of at least $2 n-1$ solutions of boundary value problem (1.1)-(1.2).

Theorem 4.1 Assume that

$\left(\mathrm{H}_{1}\right)$ there exist $n(n \in N$ and $n \geq 2)$ pairs of strict lower and upper solutions $\left\{\alpha_{i}(t)\right\}_{i=1}^{n}$, $\left\{\beta_{i}(t)\right\}_{i=1}^{n}$ of the problem (1.1)-(1.2) such that

$$
\alpha_{1}(t)<\beta_{1}(t)<\alpha_{2}(t)<\beta_{2}(t)<\cdots<\alpha_{n}(t)<\beta_{n}(t), \quad \forall t \in J
$$


$\left(\mathrm{H}_{2}\right) f: J \times R^{2} \rightarrow R, f(t, u, p)$ is continuous on $J^{*} \times R^{2}$ and has property $(H)$ relative to $\alpha_{1}(t)$, $\beta_{n}(t)$;

$\left(\mathrm{H}_{3}\right) I_{k}, J_{k}$ are continuous for each $k=1,2, \ldots, m$, and satisfy

$$
\begin{aligned}
& I_{k}\left(t_{k}, \alpha_{i}\left(t_{k}\right)\right)=I_{k}\left(t_{k}, \beta_{i}\left(t_{k}\right)\right)=0, \quad \text { and } \\
& J_{k}\left(t_{k}, \alpha_{i}\left(t_{k}\right)\right)<0<J_{k}\left(t_{k}, \beta_{i}\left(t_{k}\right)\right), \quad i=1,2, \ldots, n
\end{aligned}
$$

Then BVP (1.1)-(1.2) has at least $2 n-1$ solutions $u_{1}(t), u_{2}(t), \ldots, u_{2 n-1}(t)$ such that $\alpha_{1}(t)<$ $u_{1}(t)<\beta_{1}(t), \alpha_{2}(t)<u_{2}(t)<\beta_{2}(t), \ldots, \alpha_{n}(t)<u_{n}(t)<\beta_{n}(t)$, and

$$
\begin{aligned}
& \min _{t \in J} u_{n+1}(t)<\alpha_{2}(t), \quad \max _{t \in J} u_{n+1}(t)>\beta_{1}(t), \quad \ldots, \quad \min _{t \in J} u_{2 n-1}(t)<\alpha_{n}(t), \\
& \max _{t \in J} u_{2 n-1}(t)>\beta_{n-1}(t) .
\end{aligned}
$$

Proof Choose $M>0$ large enough such that, for $i=1,2, \ldots, n$,

$$
\begin{aligned}
& f\left(t, \beta_{i}(t), 0\right)+M-\beta_{i}(t)>0, \quad M>\beta_{i}(t), \forall t \in J, \\
& f\left(t, \alpha_{i}(t), 0\right)-M-\alpha_{i}(t)<0, \quad-M<\alpha_{i}(t), \forall t \in J, \\
& J_{k}\left(t_{k}, \alpha_{i}\left(t_{k}\right)\right)-M-\alpha_{i}\left(t_{k}\right)<0<J_{k}\left(t_{k}, \beta_{i}\left(t_{k}\right)\right)+M-\beta_{i}\left(t_{k}\right), \quad k=1, \ldots, m,
\end{aligned}
$$

and let $h(u)$ be defined in (3.10), then it follows from Lemma 3.4 that we can take $d>0$ large enough such that, for all $u \in[-M, M]$,

$$
\begin{aligned}
& \min _{[0, M]} h_{1}(u)>\max \left\{\max _{t \in J}\left|\beta_{i}^{\prime}(t)\right|, \max _{t \in J}\left|\alpha_{i}^{\prime}(t)\right|, i=1,2, \ldots, n\right\}, \\
& \min _{[-M, 0]} h_{2}(u)>\max \left\{\max _{t \in J}\left|\beta_{i}^{\prime}(t)\right|, \max _{t \in J}\left|\alpha_{i}^{\prime}(t)\right|, i=1,2, \ldots, n\right\} .
\end{aligned}
$$

Define the set $G_{i}(i=1,2, \ldots, n)$ as

$$
G_{i}=\left\{u \in P C^{1}(J)\left|(t, u, p): \alpha_{i}(t)<u<\beta_{i}(t),\right| p \mid<h(u), \forall t \in J\right\} .
$$

Also

$$
G_{n+1}=\left\{u \in P C^{1}(J)\left|(t, u, p): \alpha_{1}(t)<u<\beta_{n+1}(t),\right| p \mid<h(u), \forall t \in J\right\} .
$$

For $i=1,2, \ldots, n+1$, we define the sets

$$
\Omega_{i}=\left\{u \in P C^{1}(J):\left(t, u(t), u^{\prime}(t)\right) \in G_{i}, t \in J ;\left(t_{k}^{+}, u\left(t_{k}^{+}\right), u^{\prime}\left(t_{k}^{+}\right)\right) \in G_{i}, k=1, \ldots, m\right\} .
$$

Then by Lemma 3.4, we have

$$
\operatorname{Deg}\left[(L, N), \Omega_{i}\right]=-1, \quad i=1,2, \ldots, n+1 .
$$

From the additive property of coincidence degree, we obtain

$$
\operatorname{Deg}\left[(L, N), \Omega_{n+1} \backslash \overline{\Omega_{1} \cup \Omega_{2} \cup \cdots \cup \Omega_{n}}\right]=n-1 \geq 1 .
$$


Since $n \geq 2$ is arbitrary, we first deal with the case $n=2$, the above discussion implies that the equation $L u=N u$, that is, the problem (1.1)-(1.2) has at least one solution in the set $\Omega_{1}, \Omega_{2}$ and $\Omega_{3} \backslash \overline{\Omega_{1} \cup \Omega_{2}}$ respectively. That is, there exist at least three different solutions $u_{1}(t), u_{2}(t)$ and $u_{3}(t)$ such that

$$
\alpha_{1}(t)<u_{1}(t)<\beta_{1}(t), \quad \alpha_{2}(t)<u_{2}(t)<\beta_{2}(t), \quad \text { on } J,
$$

and $\min _{t \in J} u_{3}(t)<\alpha_{2}(t), \max _{t \in J} u_{3}(t)>\beta_{1}(t)$.

For $n=3$, replacing $\alpha_{1}, \alpha_{2}, \beta_{1}, \beta_{2}$ by $\alpha_{2}, \alpha_{3}, \beta_{2}, \beta_{3}$ respectively, then we can obtain another two different solutions $u_{4}(t), u_{5}(t)$ of the problem (1.1)-(1.2) such that

$$
\alpha_{3}(t)<u_{4}(t)<\beta_{3}(t), \quad \text { and } \quad \min _{t \in J} u_{5}(t)<\alpha_{3}(t), \quad \max _{t \in J} u_{5}(t)>\beta_{2}(t) .
$$

Along this way, we can complete the proof by the induction method.

We now present an example to illustrate that the assumptions of our theorem can be verified.

Example 1 Consider the following boundary value problem:

$$
\left\{\begin{aligned}
&\left(\rho(t) u^{\prime}\right)^{\prime}=a-2 a \sin 2 \pi u(t) \cos 4 \pi u(t)+b t^{2} \\
&+\frac{1}{2}\left(u^{\prime}\right)^{2}\left(1+\sin ^{2} u^{\prime}\right), \quad t \in[0,1], t \neq t_{k}, \\
& \Delta u\left(t_{k}\right)=\left(t_{k}-u\left(t_{k}\right)\right) \cos 2 \pi u\left(t_{k}\right), \quad k=1, \ldots, m, \\
& \Delta u^{\prime}\left(t_{k}\right)= \frac{t_{k}}{16} \operatorname{tg} \pi u\left(t_{k}\right), \quad k=1, \ldots, m, \\
& u^{\prime}(0)=0, \quad u(1)=u(\eta),
\end{aligned}\right.
$$

where $\rho(t)=c+\sin \pi t, c>1,0<b<a \leq \frac{1}{12}, 0<\eta<1,0<t_{1}<t_{2}<\cdots<t_{m}<1, \eta \neq t_{k}$.

First, we have $\rho(t) \geq c-1>0$ and $\left|\rho^{\prime}(t)\right|=|\cos t| \leq 1$, thus $\theta=1, \delta=c-1$.

Next, it is clear that $\left\{\alpha_{i}\right\}_{i=1}^{n}=\left\{-\frac{1}{4}, \frac{3}{4}, \ldots, n-\frac{5}{4}\right\}$ and $\left\{\beta_{i}\right\}_{i=1}^{n}=\left\{\frac{1}{4}, \frac{5}{4}, \ldots, n-\frac{3}{4}\right\}$ are $n(n \in N$ and $n \geq 2$ ) pairs of strict lower and upper solutions of the problem (4.1), respectively. It can be seen that $I_{k}\left(t_{k}, \alpha_{i}\left(t_{k}\right)\right)=I_{k}\left(t_{k}, \beta_{i}\left(t_{k}\right)\right)=0, J_{k}\left(t_{k}, \alpha_{i}\left(t_{k}\right)\right)<0, J_{k}\left(t_{k}, \beta_{i}\left(t_{k}\right)\right)>0, i=1, \ldots, n$. Thus conditions $\left(\mathrm{H}_{1}\right)$ and $\left(\mathrm{H}_{3}\right)$ of Theorem (4.1) hold.

Since $f(t, u, p)=a-2 a \sin 2 \pi u \cos 4 \pi u+b t^{2}+\frac{1}{2} p^{2}\left(1+\sin ^{2} p\right)$, it follows that

$$
\begin{aligned}
f\left(t, i-\frac{5}{4}, 0\right) & =a-2 a \sin \left(2 \pi\left(i-\frac{5}{4}\right)\right) \cos \left(4 \pi\left(i-\frac{5}{4}\right)\right)+b t^{2} \\
& =a-2 a+b t^{2} \leq b-a<0, \quad i=1, \ldots, n . \\
f\left(t, i-\frac{3}{4}, 0\right) & =a-2 a \sin \left(2 \pi\left(i-\frac{3}{4}\right)\right) \cos \left(4 \pi\left(i-\frac{3}{4}\right)\right)+b t^{2} \\
& =a+2 a+b t^{2}>0, \quad i=1, \ldots, n .
\end{aligned}
$$

For all $u \in\left(-\left(n-\frac{5}{4}\right),-\frac{1}{4}\right) \cup\left(\frac{1}{4},\left(n-\frac{5}{4}\right)\right)$, we have

$$
|f(t, u, p)| \leq 3 a+b t^{2}+p^{2} \leq 3 a+b+p^{2}<\frac{1}{3}+p^{2} .
$$


Take $\psi(p)=\frac{1}{3}+p^{2}$, then

$$
\int_{0}^{+\infty} \frac{s}{\theta s+\psi(s)} d s=\int_{0}^{+\infty} \frac{s}{s+\frac{1}{3}+s^{2}} d s=+\infty
$$

Hence $f$ has property $(H)$. It follows from Theorem 4.1 that the problem (4.1) has at least $2 n-1$ different solutions $u_{1}(t), \ldots, u_{n}(t), \ldots, u_{2 n-1}(t)$ such that

$$
\begin{aligned}
& -\frac{1}{4}<u_{1}(t)<\frac{1}{4}, \quad \frac{3}{4}<u_{2}(t)<\frac{5}{4}, \quad \ldots, \quad\left(n-\frac{5}{4}\right)<u_{n}(t)<\left(n-\frac{3}{4}\right), \quad \text { and } \\
& \min _{t \in J} u_{n+1}(t)<\frac{3}{4}, \quad \max _{t \in J} u_{n+1}(t)>\frac{1}{4}, \quad \ldots, \quad \min _{t \in J} u_{2 n-1}(t)<n-\frac{5}{4}, \\
& \max _{t \in J} u_{2 n-1}(t)>n-\frac{7}{4} .
\end{aligned}
$$

\section{Competing interests}

The authors declare that they have no competing interests.

\section{Authors' contributions}

The authors declare that the study was realized in collaboration with the same responsibility. All authors read and approved the final manuscript.

\section{Author details}

${ }^{1}$ School of Science, Hunan University of Technology, Zhuzhou, Hunan 412007, PR China. ${ }^{2}$ Department of Mathematics, Central South University, Changsha, Hunan 410075, PR China.

\section{Acknowledgements}

The authors are highly grateful to the referees for careful reading and comments on this paper. The research is supported by Hunan Provincial Natural Science Foundation of China (No. 13JJ3106, and 12JJ2004); it is also supported by the National Natural Science Foundation of China (No. 61074067, 11271372, and 11201138).

\section{Received: 20 December 2013 Accepted: 8 April 2014 Published: 07 May 2014}

\section{References}

1. Bainov, DD, Simeonov, PS: Systems with Impulse Effect. Ellis Horwood, Chichester (1989)

2. Lakshmikantham, V, Bainov, DD, Simeonov, PS: Theory of Impulsive Differential Equations. World Scientific, Singapore (1989)

3. Guo, D: Multiple solutions for $n$-th order impulsive integro-differential equations in Banach spaces. Nonlinear Anal. $60,955-976(2005)$

4. Liu, X, Jiang, D: Multiple positive solutions of Dirichlet boundary value problems for second order impulsive differential equations. J. Math. Anal. Appl. 321, 501-514 (2006)

5. Ding, W, Han, M, Mi, J: Periodic boundary value problems for second-order impulsive functional equations. Comput. Math. Appl. 50, 491-507 (2005)

6. Liu, B, Yu, J: Existence of solution for $m$-point boundary value problem of second order differential systems with impulsive. Appl. Math. Comput. 125, 155-175 (2002)

7. O'Regan, D: Boundary value problem for second and higher order differential equation. Proc. Am. Math. Soc. 113 , 761-775 (1991)

8. Jiang, F, Shen, J, Zeng, Y: Applications of the Poincaré-Birkhoff theorem to impulsive Duffing equations at resonance. Nonlinear Anal., Real World Appl. 13, 1292-1305 (2012)

9. Lee, Y, Liu, X: Study of singular for second order impulsive differential equations. J. Math. Anal. Appl. 331, 159-176 (2007)

10. Bai, C: Existence result for boundary value problem of nonlinear impulsive fractional differential equation at resonance. J. Appl. Math. Comput. 39, 421-443 (2012)

11. Feng, W, Webb, JRL: Solvability of three point boundary value problem at resonance. Nonlinear Anal. 30, 3227-3238 (1997)

12. Khan, RA, Webb, JRL: Existence of at least three solutions of a second-order three-point boundary value problem. Nonlinear Anal. 64, 1356-1366 (2006)

13. Ma, R: Existence results of a m-point boundary value problem at resonance. J. Math. Anal. Appl. 294, 147-157 (2004)

14. Gupta, P, Ntouyas, S, Tsamatos, P: Existence results for multi-point boundary value problem for second-order ordinary differential equations. Bull. Greek Math. Soc. 43, 105-123 (2000)

15. Henderson, J, Thompson, HB: Existence of multiple solutions for second-order boundary value problems. J. Differ. Equ. $166,443-454(2000)$ 
16. Du, Z, Xue, C, Ge, W: Multiple solutions for three-point boundary value problems nonlinear terms depending on the first order derivate. Arch. Math. 84, 341-349 (2005)

17. Du, Z, Liu, W, Lin, X: Multiple solutions to a three-point boundary value problem for higher order ordinary differential equations. J. Math. Anal. Appl. 335, 1207-1218 (2007)

18. Yang, X: Multiple periodic solutions for a class of second order differential equations. Appl. Math. Lett. 18, 91-99 (2004)

19. Zhao, Y, Chen, H: Multiplicity of solutions to two-point boundary value problems for second-order impulsive differential equations. Appl. Math. Comput. 206, 925-931 (2008)

20. Khan, RA: Generalized approximations and rapid convergence of solutions of $m$-point boundary value problems. Appl. Math. Comput. 188, 1878-1890 (2007)

21. Mawhin, J: Topological Degree Methods in Nonlinear Boundary Value Problems. CBMS, Regional Conf. Series in Math (1979)

10.1186/1687-2770-2014-103

Cite this article as: Zhao et al.: Multiple solutions of three-point boundary value problems for second-order impulsive differential equation at resonance. Boundary Value Problems 2014, 2014:103

\section{Submit your manuscript to a SpringerOpen ${ }^{\circ}$ journal and benefit from:}

- Convenient online submission

- Rigorous peer review

- Immediate publication on acceptance

- Open access: articles freely available online

- High visibility within the field

- Retaining the copyright to your article 